\title{
Breast Cancer Screening in the United Arab Emirates: Is it Time to Call for a Screening at an Earlier Age?
}

\author{
Humaid $\mathrm{O}$ Al Shamsi ${ }^{1 *}$ and Sadir Alrawi ${ }^{2}$ \\ ${ }^{1}$ MD, Department of Medicine, University of Sharjah, Sharjah, United Arab Emirates \\ ${ }^{2}$ Alzahra Hospital, Dubai, United Arab Emirates
}

Received: 䠊June 04, 2018; Published: 眥 June 18, 2018

*Corresponding author: Humaid O Al-Shamsi, MD, MRCP (UK), FRCP(C), FACP, Department of medicine , University of Sharjah, Sharjah

\section{Mini Review}

Cancer is a major health issue around the world, in the United Arab Emirates ( UAE ) it is the 3rd leading cause of death after cardiovascular diseases and injuries, accounting for $10 \%$ of all mortalities in the UAE in 2010 [1] and 16\% of all mortalities in the Emirate of Abu Dhabi in 2015 [2]. Breast cancer (BC) is the most frequent cancer in females among UAE citizens $(32.16 \%)$ as well as Non- UAE citizens (41.41\%) [3]. Females in the UAE has the tendency to develop $\mathrm{BC}$ at least a decade earlier than their counterparts in western countries [3-5]. The UAE government has made strident efforts to promote early cancer detection through different health authorities in the UAE. The Health Authority of Abu Dhabi (The UAE's largest health district) began to have screening programs for colorectal, breast, and cervical cancers in 2008 [6].
Dubai Health Authority (the second-largest health district in UAE) introduced breast, cervical, and colon cancer screening in 2014 [7]. In September 2015 [8], the Ministry of Health (covering the rest of the UAE health districts) launched an official cancer screening initiative, to raise awareness about cancer and about the importance of early detection through regular medical checkups for colorectal, breast, and cervical cancers [8,9] All the abovementioned screening efforts were aligned with the North American and European international guidelines regarding the age of onset and the frequency of screening of different cancers, including breast cancer screening $[10,11]$. Mammography screening every 2 years has shown the greatest mortality reduction benefit in the age group of 50-69 years, and it is recommended by the European Union and numerous individual countries [12].

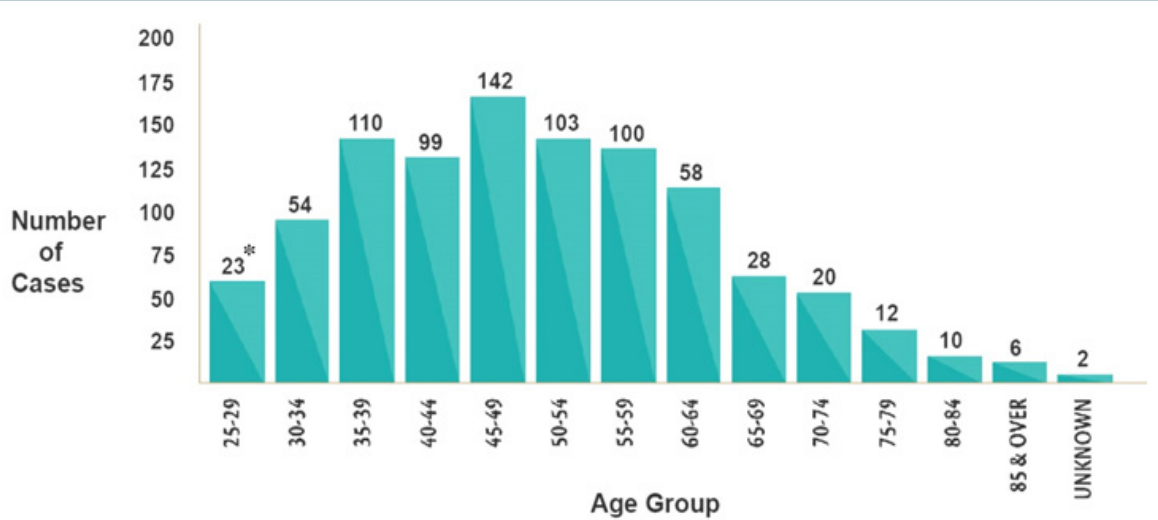

*Indicates number of cases

Figure 1: The distribution of new breast cancer cases a cross the age groups in 2014 in the United Arab Emirates.

Interestingly, Breast Cancer (BC) in the Arab population (including the UAE population) has different clinic-pathological and immune-histo chemical features than it does in the Western population, including early onset, higher grade, higher HER2 amplification rate, and lower luminal subtype $[4,13]$. It has been reported that the average age at presentation of breast cancer is a decade younger in Arab women than in European and US individuals [5]. In Arab populations, the median age at diagnosis is about 48 years, and about two-thirds of women with breast cancer are younger than 50 years $[3,5]$. The Statistics and Research Center 
under the Ministry of Health in the UAE has published the first comprehensive annual national incidence report in January 2018, for the year of 2014 [14]. The data came from the UAE National Cancer Registry (UAE-NCR), which recorded demographic, cancer, staging, clinical, and treatment information for all cancers diagnosed in UAE, in accordance with internationally-accepted registration and coding standards. For UAE and Non- UAE citizens, all malignant and in-situ cases diagnosed in UAE during 1st Jan - 31st December 2014 were notified and registered to the UAE national cancer registry. In this report, $\mathrm{BC}$ was the most common cancer in females, representing $38.8 \%$ of all malignant cases in 2014. There were 768 new cases of invasive breast cancer in the UAE in 2014.429 cases (55.8\%) were in women under the age of 50 There were 165 (21.5\%) cases of women between the age of 30 and 40 who were diagnosed with BC (Figure 1). The highest frequency of breast cancer cases were loco regional (29.4\%) followed by localized (26.4\%) followed by distant metastases (10.1\%), in situ cases were $6.2 \%$ and $27.9 \%$ were unknown stage. The age adjusted $\mathrm{BC}$ mortality was not reported [3].

Literature shows limited knowledge regarding genetic predisposition of breast cancer in Arab women but like other countries, breast cancer was found to be the most prevalent cancer in females in UAE as reported in 2004 [15]. This trend is easily visible in the recent data just published from the 2014 as mentioned earlier. Clinically, we have been observing an increasingly younger age of onset (less than 40 years of age) of BC in the UAE population (both UAE citizens and non-citizens). The concern of early-onset breast cancer in UAE has been discussed heavily among the UAE oncology community, We coined the term "Sunshine phenomenon" to described it. The unique features of $\mathrm{BC}$ in the Arab population has been described earlier $[5,13]$, yet the younger onset of $\mathrm{BC}$ in the UAE population has not been formally evaluated or reported in the literature before.

With the earlier onset of breast cancer in the female UAE population compared with the female Western population [3], it is paramount to address the current recommended starting age of screening for BC. $21.5 \%$ of the BC cases diagnosed in 2014 were between the age of 30 and 40 , That is translate to one in five $\mathrm{BC}$ cases to be diagnosed with BC under the age of 40 . This age group is not currently included in the recommendations for BC screening and is unlikely to be offered screening due to the younger age, unless there is a significant family history. Routine mammographic screening at an earlier age than the current recommended starting age of 40 years of age is an issue that has been discussed previously in the literature for high-risk group of 30- to 39-year-old black women yet it has not been implemented [16].

We are in agreement with the Najjar et al. [5] that earlier screening in Arab population is an important issue that a consensus recommendations from the local Arab oncology communities and societies are needed to address this very critical issue. The current screening recommendations in UAE have to be adjusted based on the UAE local data rather than on international and foreign data, as the UAE breast cancer population is likely different (as outlined above); utilizing international screening guidelines may miss a significant number of patients who are not eligible for screening based on these guidelines.

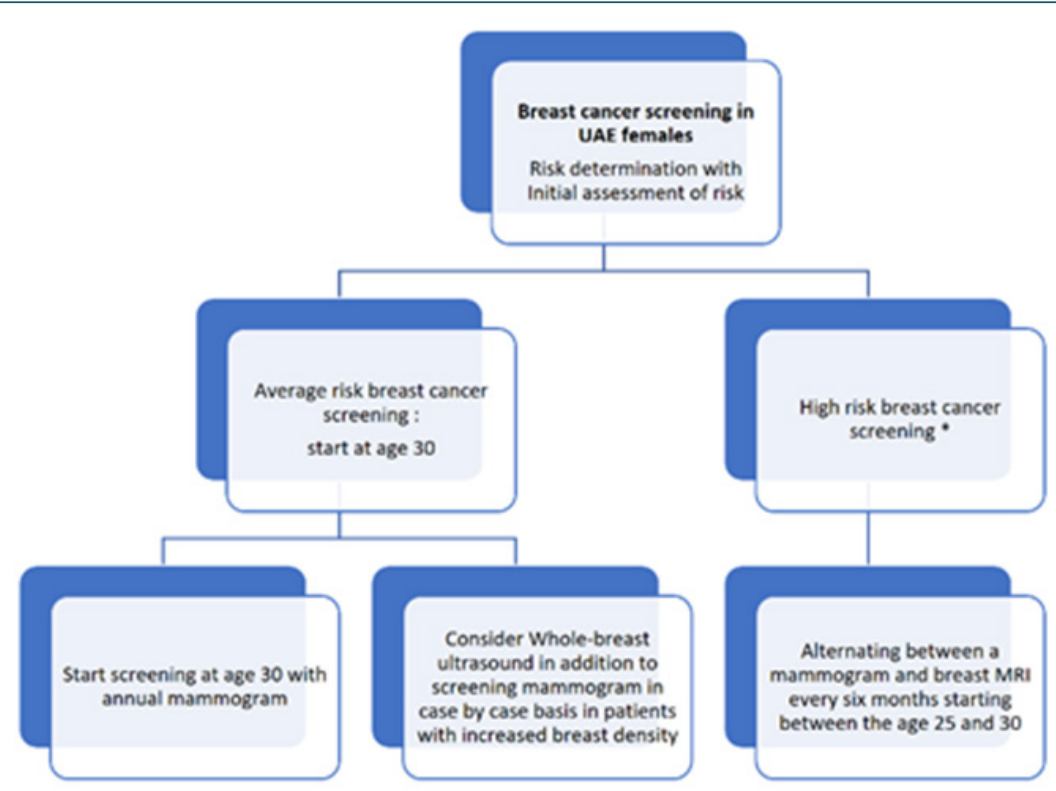

*High risk breast cancer screening if any of the following:

a. Personal history of ovarian, peritoneal (including tubal), or breast cancer,

b. Family history of breast, ovarian, or peritoneal cancer,

c. Genetic predisposition (if the patient's BRCA or other genetic marker status is known),

d. Radiotherapy to the chest between age 10 and age 30 .

Figure 2: Proposed Breast cancer screening in UAE females according to risk assessment 
Although mammography remains the preferred screening modality, Among younger women than 40, there are no randomized trials of breast cancer screening, and performance characteristics of mammography are poor. The sensitivity and specificity of mammography are also age-dependent, being higher in older women than in younger women [17]. In a review of results of 73,335 initial screening mammograms in women aged 35 to 39 years, the positive predictive value was low at only 1.3 percent [18], yet these studies are from western population and extrapolating these data to our population is again questionable. We acknowledge the limitations of mammograms in younger age groups [18], including low sensitivity [19], high breast density [19], cost/benefit concerns, and low positive predictive value [9].we also acknowledge that the evidence of mammography screening effectiveness in western women aged 40-49 years is limited; however, given the unique clinical presentation of our population and also in the light of the presented data above, we argue for considering early screening mammography starting at the age of 30 years in spite of recognized concerns to offer screening at this earlier age. We acknowledge the risk of over-diagnosis and over-investigations with breast cancer screening at this earlier age; yet, until further data from our region is available, we believe this is the most appropriate action at the current time and with the limited available data, in order to reduce the burden of $\mathrm{BC}$ in younger patient populations in UAE (Figure 2).

One of the concerns regarding the screening of younger population is the increase of breast density which is inversely related to patient age, and dense breasts are most commonly associated with women under age 50 or who are premenopausal [20]. Some may suggest to consider Whole-breast ultrasound screening as supplemental tool in addition to the mammography to overcome the increased breast density. Whole-breast ultrasound can detect early-stage breast cancers that are mammographic ally occult in women with dense breast tissue, but the additional screening test carries a substantial risk for false-positive results. When used as a supplement to mammography, ultrasound can improve the sensitivity of screening at the expense of decreased specificity and an increased breast biopsy rate, leading to patient anxiety, breast biopsies with benign results, and follow-up imaging [21]. The addition of ultrasound to mammographic screening has yet to be shown to reduce breast cancer mortality [22]. There have been no randomized trials comparing screening with supplemental ultrasound and mammography and screening with mammography alone in average-risk women [23]. It is imperative to acknowledge there is evidence that interpreting breast density may be somewhat subjective, with variation across different radiologists exist [15]. We suggest to consider supplemental whole-breast ultrasound in addition to screening mammogram in case by case basis in screening of younger patients with increased breast density.

Some may argue to consider screening with MRI, yet screening MRI is not recommended for average-risk women regardless of the age according to supplemental screening MRI guidelines from the
American Cancer Society guidelines for breast screening with MRI as an adjunct to mammography, MRI performed in combination with mammography is used primarily to screen high-risk patients [24]. In a recent large population-based cohort including 812,164 women undergoing breast cancer screening with mammography vs MRI with or without mammography with a total of 2,048,994 digital mammography and/or breast MRI screening episodes during the study period, there were two fold and five fold higher core and surgical biopsy rates following MRI compared with mammography among women with and without a personal history of breast cancer implying false-positives with MRI, respectively. There was a higher biopsy rates coupled with significantly lower cancer yield findings following biopsy compared with screening mammography alone. Another approach which can also be considered is to focus early screening starting at the age of 30 in high risk population with family history of breast cancer, yet it could be challenging to identify this high risk population which could is eligible for early breast screening. Challenges could include, failure of health providers to recognise high risk population, lack of awareness of patients about their high risk status as in many cases patients are not aware of their family history since many patients may not disclose their cancer diagnosis to their own families which is not uncommon in the Arab society. This is due to the fear of stigma and believing that not sharing the diagnosis is protective to their families. Another important aspect of the issue is the insurance coverage for the screening of privately-insured individuals (not covered by government-funded insurance), a process which is currently not standardized and which can provide challenges in getting approval for approved cancer screening tests. Currently, there are no health authority-approved guidelines for insurance companies to follow and comply with. This needs to be addressed by including approved cancer screening tests as a mandatory coverage for eligible patients, which needs to be outlined by health authorities including a definition of it is deemed to be approved cancer screening tests. Adjusting the screening age for BC screening in the local screening guideline will be a mandatory component, in order to ensure the compliance of the payers and insurance companies with the new early recommended screening age for BC.

In summary, based on the recent data from the UAE National Cancer Registry, UAE females likely to develop BC at least a decade earlier than their counterparts in western countries, and the adoption of Western BC screening guidelines without critical amendment in planning local breast cancer programs will likely miss a significant at-risk younger populations and may not achieve the desired outcomes of reducing BC-related morbidity and mortality. Despite the limitation and challenges with mammogram screening in younger population, We believe early screening mammography starting at the age of 30 is justifiable and the most appropriate action at the current time and with the limited available data, in order to reduce the burden of $\mathrm{BC}$ in younger patient populations in the UAE. Whole-breast ultrasound in addition to screening mammogram should be considered in case by case basis in patients 
with increased breast density. Further research is need to evaluate the efficacy of screening mammogram in the UAE population with focus on younger age population.

\section{References}

1. Loney T, Aw TC, Handysides DG (2013) An analysis of the health status of the United Arab Emirates: the 'Big 4' public health issues. Glob Health Action 6:20100.

2. (2015) Dhabi HSHAA: Health Statistics Health Authority - Abu Dhabi.

3. Cancer Incidence in United Arab Emirates (2014) Annual Report of the UAE - National Cancer Registry.

4. Chouchane L, Boussen H, Sastry KS (2013) Breast cancer in Arab populations: molecular characteristics and disease management implications. Lancet Oncol 14(10): e417-e424.

5. Najjar H, Easson A (2010) Age at diagnosis of breast cancer in Arab nations. Int J Surg 8(6): 448-452.

6. (2018) HAAD Cancer Programs.

7. (2014) Breast cancer screening available at DHA.

8. Ministry of Health launches (2018) The National Periodic Health Screening' and 'Cancer Screening' initiatives.

9. Chen TH, Yen AM, Fann JC (2017) Clarifying the debate on populationbased screening for breast cancer with mammography: A systematic review of randomized controlled trials on mammography with Bayesian meta-analysis and causal model. Medicine (Baltimore) 96(3): e5684.

10. Oeffinger KC, Fontham ET, Etzioni R (2015) Breast Cancer Screening for Women at Average Risk: 2015 Guideline Update From the American Cancer Society. JAMA 314(15): 1599-1614.

11. Senkus E, Kyriakides S, Penault Llorca F (2013) Primary breast cancer: ESMO Clinical Practice Guidelines for diagnosis, treatment and followup. Ann Oncol 24(Suppl 6): vi7-vi23.

12. Association of European Cancer Leagues. European Union Council Recommendation on Cancer Screening.
13. Al Tamimi DM, Shawarby MA, Ahmed A (2010) Protein expression profile and prevalence pattern of the molecular classes of breast cancer-a Saudi population based study. BMC Cancer 10: 223.

14. Coley WB (1991) The treatment of malignant tumors by repeated inoculations of erysipelas: with a report of ten original cases1893. Am J Med Sci 105: 487.

15. Sprague BL, Conant EF, Onega T (2016) Variation in Mammographic Breast Density Assessments Among Radiologists in Clinical Practice: A Multicenter Observational Study. Ann Intern Med 165(7): 457-464.

16. Johnson ET (2002) Breast cancer racial differences before age 40--implications for screening. J Natl Med Assoc 94(3): 149-156.

17. Armstrong K, Moye E, Williams S (2007) Screening mammography in women 40 to 49 years of age: a systematic review for the American College of Physicians. Ann Intern Med 146(7): 516-526.

18. Yankaskas BC, Haneuse S, Kapp JM (2010) Performance of first mammography examination in women younger than 40 years. J Natl Cancer Inst 102(10): 692-701.

19. Kerlikowske K, Grady D, Barclay J (1996) Effect of age, breast density, and family history on the sensitivity of first screening mammography. JAMA 276(1): 33-38.

20. Burton A, Maskarinec G, Perez Gomez B (2017) Mammographic density and ageing: A collaborative pooled analysis of cross-sectional data from 22 countries worldwide. PLoS Med 14.

21. Phoebe E Freer, Priscilla J Slanetz (2018) Breast density and screening for breast cancer

22. Slanetz PJ, Freer PE, Birdwell RL (2015) Breast-density legislation-practical considerations. N Engl J Med 372(7): 593-595.

23. Gartlehner G, Thaler K, Chapman A (2013) Mammography in combination with breast ultrasonography versus mammography for breast cancer screening in women at average risk. Cochrane Database Syst Rev 4: CD009632.

24. Saslow D, Boetes C, Burke W (2007) American Cancer Society guidelines for breast screening with MRI as an adjunct to mammography. CA Cancer J Clin 57(2): 75-89.

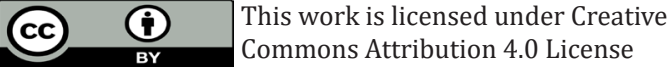

To Submit Your Article Click Here: Submit Article

DOI: $10.32474 /$ OAJOM.2018.02.000131

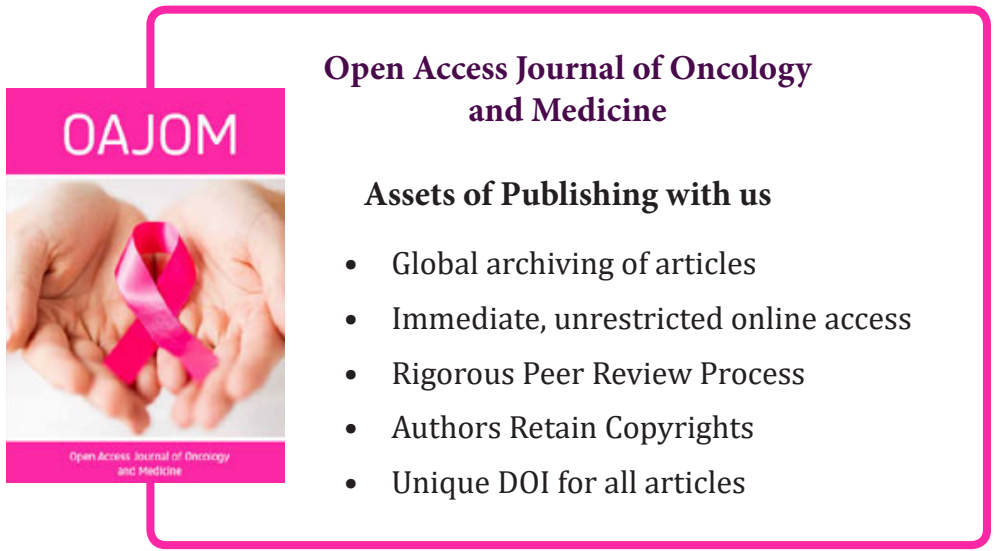

